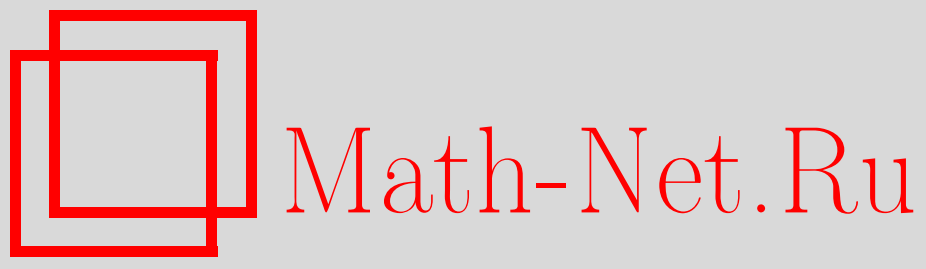

Б. И. Голубов, Модифицированный двоичный интеграл и производная дробного порядка на $\mathbb{R}_{+}$, Функи. анализ и его прил., 2005, том 39, выпуск 2, 64-70

DOI: https://doi.org/10.4213/faa41

Использование Общероссийского математического портала MathNet.Ru подразумевает, что вы прочитали и согласны с пользовательским соглашением

http://www. mathnet.ru/rus/agreement

Параметры загрузки:

IP : 3.89 .197 .203

26 апреля 2023 г., 11:57:26

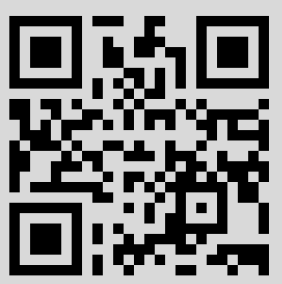


$\beta_{\infty} \leqslant \alpha_{0}$. Тогда равенство (3) верно в том и только том случае, когда выполнено (4).

В частности, рассмотрим случай степенных весовых функций $w_{0}(x)=x^{l}$ и $w_{1}(x)=x^{m}$. Тогда условия $(*)$ выполняются, если (а) $l>p_{0}-1$ и $m \leqslant 0$ или (b) $l>p_{0}-1, l>m$ и $0 \leqslant m<p_{1}-1$. Так как $k(t) \asymp t^{\theta_{0}}$, где $\theta_{0}=\frac{p_{1}\left(l-p_{0}+1\right)}{p_{1}(l+1)-p_{0}(m+1)}$, то $\alpha=\beta=\alpha_{0}=\beta_{\infty}=\theta_{0}$, и мы получаем

СлеДСТВие 3. Предположим, что для $p_{0}, p_{1}\left(1 \leqslant p_{0}<p_{1}<\infty\right), l$ u выполнено хотя бы одно из условий (a) и (b). Тогда равенство

$$
\left(L_{p_{0}}\left(x^{l}\right) \cap N, L_{p_{1}}\left(x^{m}\right) \cap N\right)_{\theta, q}=\left(L_{p_{0}}\left(x^{l}\right), L_{p_{1}}\left(x^{m}\right)\right)_{\theta, q} \cap N
$$

выполнено в том и только том случае, когда $\theta \neq \frac{p_{1}\left(l-p_{0}+1\right)}{p_{1}(l+1)-p_{0}(m+1)}$.

В случае $p_{0}=p_{1}=q$ последнее утверждение иным способом было доказано в $[1$, Corollary 1$]$.

Автор благодарен проф. Л. Малигранда за полезные обсуждения некоторых вопросов, связанных с доказательством теоремы 2.

\title{
ЛитератУра
}

1. Krugljak N., Maligranda L., Persson L.-E. Ark. Mat., 37, 323-344 (1999). 2. Ivanov S., Kalton N. Алгебра и анализ, 13, No. 2, 93-115 (2001). 3. Лионс Ж. Л., Мадженес Э. Неоднородные граничные задачи и их приложения. Мир, М., 1971. 4. Triebel H. Math. Nachr., 69, 57-60 (1975). 5. Трибель X. Теория интерполяции, функциональные пространства, дифференциальные операторы. Мир, М., 1980. 6. Maligranda L. Suppl. Rend. Circ. Mat. Palermo, 10, 113-118 (1985). 7. Wallsten R. In: Lect. Notes in Math., Vol. 1902, 1988, pp. 410-419. 8. Pisier G. Pasific J. Math., 155, 341-368 (1992). 9. Janson S. Ark. Math., 31, 307-338 (1993). 10. Кисляков С. В., Куанхуа Шу. Алгебра и анализ, 8, вып. 4, 75-109 (1996). 11. Löfström J. Interpolation of subspaces, Technical report No. 10, Univ. of Göteborg, 1997. 12. Astashkin S. V. J. Math. Math. Sci., 25, No. 7, 451-465 (2001). 13. Kaijser S., Sunehag P. Interpolation subspaces and the unit problem, In: Function Spaces, Interpolation Theory and Related Topics (Lund 2000), de Gruyter, Berlin, 2002, pp. 345-353. 14. Берг Й., Лефстрем Й. Интерполяционные пространства. Введение. Мир, М., 1980.

Самарский госуниверситет

e-mail: astashkn@ssu.samara.ru

Поступило в редакцию 28 августа 2003 г.

УДК 517.44

\section{Модифицированный двоичный интеграл и производная дробного порядка на $\mathbb{R}_{+}{ }^{*}$}

\author{
(с) 2005. Б. И. Голуьов
}

1. В работе [1] Бутцер и Вагнер ввели сильную двоичную производную и интеграл порядка $r=1,2, \ldots$ и доказали, что они являются взаимно обратными операторами. Их определения относятся к функциям $f \in L[0,1)$ или $f \in L(G)$, где $G$ - двоичная группа, изоморфная модифицированному отрезку $[0,1]^{*}$.

*Работа поддержана РФФИ, грант 05-01-00206. 
В другой работе [2] тех же авторов введена сильная двоичная производная для функций $f \in L\left(\mathbb{R}_{+}\right)$, а Вагнер [3] определил сильный двоичный интеграл также для функций $f \in L\left(\mathbb{R}_{+}\right)$, где $\mathbb{R}_{+}=[0,+\infty)$. Точечные двоичные производные $f^{[r]}(x)$ порядка $r=1,2, \ldots$ для функций $f \in L[0,1)$ и их связь с сильными интегралами $I_{r} f$ подробно изучались в работе Бутцера и Вагнера [4].

Для функций $f \in L\left(G_{p}\right)$, где $G_{p}$ - компактная группа порядка $p=2,3, \ldots$, изоморфная модифицированному отрезку $[0,1]_{p}^{*}$, понятие сильной двоичной производной и интеграла дробного порядка $\alpha>0$ введено Онневиром [5], который установил, что они являются взаимно обратными операторами. Этот же факт для $\alpha=1$ и функций, заданных на более общих компактных группах, доказали Пал и Шимон [6]. В другой работе Онневира [7] введены модифицированные точечная и сильная двоичные производные для функций на локально компактной двоичной группе $K_{2}$, изоморфной модифицированной положительной полуоси $\mathbb{R}_{+}^{*}$. Пал [8] получил достаточное условие для точечного дифференцирования в смысле Бутцера-Вагнера преобразования Фурье-Уолша.

В нашей работе [9] для функций $f \in L\left(\mathbb{R}_{+}\right)$изучались свойства операторов модифицированного двоичного сильного дифференцирования и интегрирования первого порядка.

В данной работе для функций $f \in L\left(\mathbb{R}_{+}\right)$определяются модифицированные точечные и сильные двоичные производные и интегралы положительного порядка и устанавливаются их свойства.

2. Напомним определение преобразования Фурье-Уолша, которое впервые появилось в работе Файна [10] (см. также [11, Ch. 9] и [12, гл. 6]). Для числа $x \in \mathbb{R}_{+}$и натурального $n$ положим

$$
x_{n} \equiv\left[2^{n} x\right] \quad(\bmod 2), \quad x_{-n} \equiv\left[2^{1-n} x\right] \quad(\bmod 2),
$$

где $[a]$ обозначает целую часть числа $a$, а числа $x_{n}$ и $x_{-n}$ по определению равны 0 или 1. Отметим, что $x_{n}\left(x_{-n}\right)$ для $n \in \mathbb{N}$ является $n$-м двоичным разрядом дробной (целой) части числа $x \in \mathbb{R}_{+}$, а двоично-рациональным числам $x \in \mathbb{R}_{+}$ сопоставляются конечные двоичные разложения.

Поскольку $x_{-n}=0$ для $n \geqslant n(x)$, то для $x, y \in \mathbb{R}_{+}$определено целое неотрицательное число $t(x, y)=\sum_{n=1}^{\infty}\left(x_{n} y_{-n}+x_{-n} y_{n}\right)$. Ядро Уолша $\psi(x, y)$ определяется для $(x, y) \in \mathbb{R}_{+} \times \mathbb{R}_{+}$равенством

$$
\psi(x, y)=(-1)^{t(x, y)} .
$$

Преобразование Фурье-Уолша $F[f] \equiv \tilde{f}$ функции $f \in L\left(\mathbb{R}_{+}\right)$задается равенством

$$
F[f](x) \equiv \tilde{f}(x)=\int_{\mathbb{R}_{+}} \psi(x, y) f(y) d y
$$

Это определение можно обобщить на функции из пространства $L^{p}\left(\mathbb{R}_{+}\right)$, где $1 \leqslant p \leqslant 2$, но это нам не потребуется.

Свойства преобразования Фурье-Уолша (3) аналогичны свойствам обычного преобразования Фурье (см., например, [11, ch. 9] или [12, гл. 6]).

Функциональный анализ и его приложения, т. 39, вып. 2 
На $\mathbb{R}_{+}$введем операцию сложения $\oplus$, положив $x \oplus y=z$ для $x, y \in \mathbb{R}_{+}$, где число $z$ в двоичной системе счисления имеет двоичные разряды $z_{n} \equiv x_{n}+y_{n}$ $(\bmod 2), n \in \mathbb{Z} \backslash\{0\}$, а $x_{n}, y_{n}$ вычисляются по правилу $(1)$.

Напомним определение $W$-непрерывности функции. Функцию $f: \mathbb{R}_{+} \rightarrow \mathbb{R}$ назовем $W$-непрерывной в точке $x \in \mathbb{R}_{+}$, если для любого $\varepsilon>0$ существует такое $\delta>0$, что $|f(x \oplus y)-f(x)|<\varepsilon$ при $0<y<\delta$ (см. [12, гл. 1]).

Отметим, что преобразование Фурье-Уолша $\tilde{f}$ функции $f \in L\left(\mathbb{R}_{+}\right)$является $W$-непрерывной функцией на $\mathbb{R}_{+}$(см. [12, теорема 6.1.5]), а ядро Уолша $W$-непрерывно по каждому своему аргументу при фиксированном другом аргументе.

Введем понятие двоичной точки Лебега функции. Точку $x \in \mathbb{R}_{+}$назовем двоичной точкой Лебега локально интегрируемой на $\mathbb{R}_{+}$функции $f$, если она определена в точке $x$ и $\lim _{n \rightarrow+\infty} 2^{n} \int_{0}^{2^{-n}}|f(x \oplus t)-f(x)| d t=0$. Очевидно, что точка $W$-непрерывности функции является ее двоичной точкой Лебега, а обратное утверждение неверно. Почти все точки локально интегрируемой на $\mathbb{R}_{+}$ функции $f$ являются ее двоичными точками Лебега.

3. Определим функцию $h:(0,+\infty) \rightarrow(0,+\infty)$ равенствами $h(x)=2^{-n}, 2^{n} \leqslant$ $x<2^{n+1}, n \in \mathbb{Z}$.

Лемма 1. Для $\alpha>0$ u $n \in \mathbb{Z}$ во всякой точке $x>0$ определена функиия $W_{n}^{\alpha}(x)=\lim _{m \rightarrow+\infty} \int_{2^{-n}}^{2^{m}} \psi(x, y)(h(y))^{\alpha} d y$, причем $W_{n}^{\alpha} \in L\left(\mathbb{R}_{+}\right) u W_{n}^{\alpha}(x)=0$ для $x \geqslant 2^{n}$.

Для $\alpha>0$ и $n \in \mathbb{Z}$ положим $\Lambda_{n}^{\alpha}(x)=\int_{0}^{2^{n}}(h(t))^{-\alpha} \psi(x, t) d t, x \in \mathbb{R}_{+}$.

Лемма 2. При $\alpha>0, n \in \mathbb{Z}$ справедливо включение $\Lambda_{n}^{\alpha} \in L\left(\mathbb{R}_{+}\right) \cap L^{\infty}\left(\mathbb{R}_{+}\right)$.

Напомним определение двоичной свертки $f * g$ двух функций $f, g \in L\left(\mathbb{R}_{+}\right)$:

$$
(f * g)(x)=\int_{\mathbb{R}_{+}} f(y) g(x \oplus y) d y, \quad x \in \mathbb{R}_{+} .
$$

Как и в случае обычной свертки, $f * g \in L\left(\mathbb{R}_{+}\right), \widetilde{(f * g)}=\tilde{f} \tilde{g}$.

ОПрЕДЕЛЕНИЕ 1 . Если $\alpha>0$ и для функций $f, g \in L\left(\mathbb{R}_{+}\right)$выполняется условие $\lim _{n \rightarrow+\infty}\left\|f * W_{n}^{\alpha}-g\right\|_{L\left(\mathbb{R}_{+}\right)}=0$, то функцию $g \equiv J_{\alpha}(f)$ назовем модифииированным сильным двоичным интегралом (МСДИ) порядка $\alpha$ функции $f$. Заменив в этом определении ядра $W_{n}^{\alpha}$ на $\Lambda_{n}^{\alpha}$, получим определение модифицированной сильной двоичной производной $(M C Д \Pi) g \equiv D^{(\alpha)}(f)$ порядка $\alpha$ функции $f$.

Критерий существования МСДИ (МСДП) порядка $\alpha>0$ дает

Теорема 1. Пусть $\alpha>0$ u $f, g \in L\left(\mathbb{R}_{+}\right)$. Тогда бункиия $g$ является МСДИ (соответственно МСДП) порядка а функиии $f$ тогда и только тогда, когда $\tilde{g}(0)=0$ и $\tilde{g}(x)=\tilde{f}(x)(h(x))^{\alpha}$ для $x>0$ (соответственно $\tilde{g}(0)=0$ и $\tilde{g}(x)=$ $\tilde{f}(x)(h(x))^{-\alpha}$ для $\left.x>0\right)$.

Для сильного двоичного интеграла и производной первого порядка в смысле Бутцера-Вагнера подобный результат доказан в работе [3], а вторая часть этой теоремы фактически доказана в работе [13], однако там МСДП порядка $\alpha>0$ определена несколько иначе.

Следующая теорема устанавливает связь между операторами $J_{\alpha}$ и $D^{(\alpha)}$. 
Теорема 2. Если у функиии $f \in L\left(\mathbb{R}_{+}\right)$при некотором $\alpha>0$ существуeт МСДИ $J_{\alpha}(f)$ (существует $М С Д П ~ D^{(\alpha)}(f)$ u $\left.\tilde{f}(0)=0\right)$, mо у $J_{\alpha}(f)$ (co-

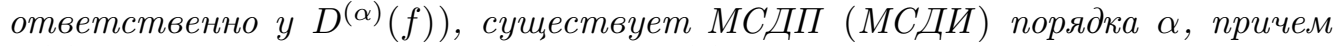
$D^{(\alpha)}\left(J_{\alpha}(f)\right)=f\left(\right.$ соответственно $\left.J_{\alpha}\left(D^{(\alpha)}(f)\right)=f\right)$.

Для сильного двоичного интеграла и производной натурального порядка в смысле Бутцера-Вагнера подобный результат известен для функций $f \in L(G)$, где $G=[0,1]$ или $G=\mathbb{R}_{+}$(см. [3] и [4]). Для функций $f \in L\left(G_{p}\right)$, где $G_{p}$ - компактная группа порядка $p=2,3, \ldots$, изоморфная модифицированному отрезку $[0,1]_{p}^{*}$, результат, подобный теореме 2, доказан Онневиром [5].

Семейство операторов $\left\{J_{\alpha}\right\}_{\alpha \geqslant 0}\left(\left\{D^{(\alpha)}\right\}_{\alpha \geqslant 0}\right)$ образует полугруппу с единицей $I \equiv J_{0}=D^{(0)}$.

Теорема 3. Пусть $\alpha, \beta \geqslant 0$ и бункиия $f \in L\left(\mathbb{R}_{+}\right)$имеет $М С Д И J_{\alpha}(f) \equiv \varphi$ $\left(M C Д \Pi D^{(\alpha)}(f) \equiv \varphi\right)$ порядка $\alpha$, а функиия $\varphi$ имеет $М С Д И ~ J_{\beta}(\varphi)(М С Д П$ $\left.D^{(\beta)}(\varphi)\right)$ порядка $\beta$. Тогда функиия $f$ имеет $М С Д И ~ J_{\alpha+\beta}(f)\left(M C Д \Pi D^{(\alpha+\beta)}(f)\right)$ порядка $\alpha+\beta$, причем $J_{\alpha+\beta}(f)=J_{\beta}\left(J_{\alpha}(f)\right)\left(\right.$ соответственно $D^{(\alpha+\beta)}(f)=$ $\left.D^{(\beta)}\left(D^{(\alpha)}(f)\right)\right)$.

Для функций $f \in L\left(G_{p}\right)$ результат, подобный второй части теоремы 2 , доказан в работе [5].

Известно, что система функций $\left\{\bar{a}_{m, n}\right\}_{m=0}^{+\infty}$, где $\bar{a}_{m, n}(x)=2^{-n / 2} \psi\left(x, m 2^{-n}\right)$ при любом $n \in \mathbb{Z}_{+}$ортонормирована на $\Delta_{n}=\left[0,2^{n}\right)$ (см. [12, теорема 1.5.6]) и полна в $L^{p}\left(\Delta_{n}\right), 1 \leqslant p \leqslant \infty[14]$.

В следующей теореме указана система собственных функций операторов $J_{\alpha}$ и $D^{(\alpha)}$, а через $\mathrm{X}_{E}(x)$ обозначена характеристическая функция множества $E \subset \mathbb{R}_{+}$.

ТеОремА 4. Каждая из функиий $a_{m, n}(x)=\psi\left(x, m 2^{-n}\right) \mathrm{X}_{\left[0,2^{n}\right)}(x)(m \in \mathbb{N}$, $n \in \mathbb{Z})$ имеет МСДИ и МСДП порядка $\alpha>0$, причем $J_{\alpha}\left(a_{m, n}\right)=2^{-r \alpha} a_{m, n}$ и $D^{(\alpha)}\left(a_{m, n}\right)=2^{r \alpha} a_{m, n}$, где челое число $r=r(m, n)$ однозначно определяется вложсением $\left[m 2^{-n},(m+1) 2^{-n}\right) \subset\left[2^{r}, 2^{r+1}\right)$.

ЗАмЕЧАНИЕ 1. Для функций $a_{0, n}(x)=\mathrm{X}_{\left[0,2^{n}\right)}(x)(n \in \mathbb{Z})$ не существует МСДИ порядка $\alpha \geqslant 1$.

СлЕДСТВИЕ 1. При $\alpha>0$ справедливы равенства $J_{\alpha}\left(a_{1, n}\right)=2^{n \alpha} a_{1, n}, n \in \mathbb{Z}_{+}$, $u D^{(\alpha)}\left(a_{m, 0}\right)=2^{r \alpha} a_{m, 0}, 2^{r} \leqslant m<2^{r+1}$, где $r=0,1, \ldots$.

Обозначим через $L_{J_{\alpha}}\left(L_{D^{(\alpha)}}\right)$ множество всех функций $f \in L\left(\mathbb{R}_{+}\right)$, для каждой из которых существует МСДИ $J_{\alpha}(f)\left(\mathrm{MCДП} D^{(\alpha)}(f)\right)$. Очевидно, что $L_{J_{\alpha}}$ и $L_{D^{(\alpha)}}$ являются линейными подпространствами в $L\left(\mathbb{R}_{+}\right)$.

СЛЕДСТВИЕ 2. Линейные операторъ $J_{\alpha}: L_{J_{\alpha}} \rightarrow L\left(\mathbb{R}_{+}\right)$u $D^{(\alpha)}: L_{D^{(\alpha)}} \rightarrow$ $L\left(\mathbb{R}_{+}\right)$не ограничены при любом $\alpha>0$.

СЛЕДСТВИЕ 3. При любом $\alpha>0$ оба множества $L_{J_{\alpha}} u L_{D^{(\alpha)}}$ nлотны $в$ двоччном пространстве Харди $H\left(\mathbb{R}_{+}\right)$(определение пространства $H\left(\mathbb{R}_{+}\right)$дано в работе [15]).

Отметим, что существование сильного двоичного интеграла первого порядка в смысле Бутцера-Вагнера от функций $a_{m, n}(x)=\psi\left(x, m 2^{-n}\right) \mathrm{X}_{\left[0,2^{n}\right)}(x)(m \in \mathbb{N}$, $n \in \mathbb{Z})$ установлено в работе Пала и Шиппа [16]. В ней доказано, что линейная 
оболочка этой системы функций плотна в метрике пространства $L\left(\mathbb{R}_{+}\right)$в множестве всех функций, имеющих сильный двоичный интеграл первого порядка в смысле Бутцера-Вагнера.

4. Введем модифицированные точечные двоичные производные и интегралы дробного порядка.

ОПРЕДЕЛЕНИЕ 2 . Если $f \in L\left(\mathbb{R}_{+}\right)$и при некотором $\alpha>0$ в точке $x \in \mathbb{R}_{+}$существует конечный предел $d^{(\alpha)}(f)(x) \equiv \lim _{n \rightarrow+\infty} \int_{0}^{2^{n}}(h(y))^{-\alpha} \tilde{f}(y) \psi(x, y) d y$, то назовем его модифицированной двоичной производной (МДП) порядка $\alpha$ функции $f$ в точке $x$. Если же существует конечный предел

$$
j_{\alpha}(f)(x) \equiv \lim _{n \rightarrow+\infty} \int_{2^{-n}}^{2^{n}}(h(y))^{\alpha} \tilde{f}(y) \psi(x, y) d y,
$$

то этот предел назовем модифицированным двоичным интегралом (МДИ) порядка $\alpha$ функции $f$ в точке $x$.

Достаточные условия существования МДП и МДИ порядка $\alpha>0$ дает

Tеорема 5. Если $\alpha>0$, а функиия $f \in L\left(\mathbb{R}_{+}\right)$такова, что $h^{-\alpha} \tilde{f} \in L\left(\mathbb{R}_{+}\right)$ $\left(h^{\alpha} \tilde{f} \in L\left(\mathbb{R}_{+}\right)\right)$, то существует $W$-непрерывная МДП $d^{(\alpha)}(f)(x)$ (W-непрерывный МДИ $\left.j_{\alpha}(f)(x)\right)$ на $\mathbb{R}_{+}$, причем $d^{(\alpha)}(f)(x)=\int_{\mathbb{R}_{+}}(h(y))^{-\alpha} \tilde{f}(y) \psi(x, y) d y$ $\left(j_{\alpha}(f)(x)=\int_{\mathbb{R}_{+}}(h(y))^{\alpha} \tilde{f}(y) \psi(x, y) d y\right)$.

Следующую теорему можно считать двоичным аналогом классических теорем Лебега о дифференцировании интеграла Лебега по его верхнему пределу и о восстановлении функции по ее производной.

Теорема 6. Пусть $\alpha>0$, а функиия $f \in L\left(\mathbb{R}_{+}\right)$имеет МСДИ $J_{\alpha}(f)$ $\left(M C Д П D^{(\alpha)}(f)\right)$ порядка $\alpha$. Тогда во всякой двоччной точке Лебега $x \in \mathbb{R}_{+}$ функиии $f$, а следовательно, почти всюду на $\mathbb{R}_{+}$справедливо равенство $d^{(\alpha)}\left(J_{\alpha}(f)\right)(x)=f(x)\left(j_{\alpha}\left(D^{(\alpha)}(f)\right)(x)=f(x)\right)$.

Отметим, что для функций $f \in L[0,1]$ при условии $\int_{0}^{1} f(x) d x=0$ и натуральных $r$ справедливо равенство $\left(I_{r} f\right)^{[r]}(x)=f(x)$ почти всюду, где $I_{r} f-$ сильный интеграл $\left(f^{[r]}\right.$ - двоичная точечная производная) порядка $r$ в смысле Бутцера-Вагнера. Это доказал Шипп [17], отвечая на вопрос Вагнера [18]. В работе [4] доказан критерий того, что почти всюду на [0, 1] справедливо равенство $f(x)=\int_{0}^{1} f(t) d t+\left(I_{r} f^{[r]}\right)(x)$, где $r$ натуральное. Для функций $f \in L\left(\mathbb{R}_{+}\right)$, имеющих интеграл первого порядка $I_{1} f$ в смысле Бутцера-Вагнера, равенство $\left(I_{1} f\right)^{[1]}(x)=f(x)$ почти всюду доказали Пал и Шипп (см. [19] или [11, p. 445]).

Сильные и точечные двоичные производные (интегралы) согласованы друг с другом.

Теорема 7. Если у функции $f \in L\left(\mathbb{R}_{+}\right)$существует $M С Д П ~ D^{(\alpha)}(f)(М С Д И$ $\left.J_{\alpha}(f)\right)$ порядка $\alpha>0$ и $h^{-\alpha} \tilde{f} \in L\left(\mathbb{R}_{+}\right)\left(h^{\alpha} \tilde{f} \in L\left(\mathbb{R}_{+}\right)\right)$, то у функиии $f$ в кажсдой точке $x \in \mathbb{R}_{+}$существует МДП $d^{(\alpha)}(f)(x)$ (существует МДИ $j_{\alpha}(f)(x)$ ) порядка $\alpha$, причем $D^{(\alpha)}(f)(x)=d^{(\alpha)}(f)(x)\left(\right.$ соответственно $\left.J_{\alpha}(f)(x)=j_{\alpha}(f)(x)\right)$ почти всюду на $\mathbb{R}_{+}$.

Отметим, что для ядер $\Lambda_{n}^{\alpha}$ и $W_{n}^{\alpha}$ справедливы включения $\Lambda_{n}^{\alpha} \in L\left(\mathbb{R}_{+}\right) \cap$ $L^{\infty}\left(\mathbb{R}_{+}\right)$и $W_{n}^{\alpha} \in L\left(\mathbb{R}_{+}\right)$при $n \in \mathbb{Z}, \alpha>0$ (см. леммы 1 и 2 ). Поэтому свертка $\left(f * \Lambda_{n}^{\alpha}\right)(x)$ определена в каждой точке $x \in \mathbb{R}_{+}$для функций $f \in L\left(\mathbb{R}_{+}\right) \cup$ 
$L^{\infty}\left(\mathbb{R}_{+}\right)$, а свертка $\left(f * W_{n}^{\alpha}\right)(x)$ - для функций $f \in L^{\infty}\left(\mathbb{R}_{+}\right)$. Этот факт позволяет ввести следующее

ОПРЕДЕЛЕНИЕ 3. Если $\alpha>0$ и для функции $f \in L\left(\mathbb{R}_{+}\right) \cup L^{\infty}\left(\mathbb{R}_{+}\right)$в точке $x \in \mathbb{R}_{+}$существует конечный предел $\bar{d}^{(\alpha)}(f)(x)=\lim _{n \rightarrow+\infty}\left(f * \Lambda_{n}^{\alpha}\right)(x)$, то число $\bar{d}^{(\alpha)}(f)(x)$ назовем двоичной производной (ДП) порядка $\alpha$ функции $f$ в точке $x$. Если же для функции $f \in L^{\infty}\left(\mathbb{R}_{+}\right)$в точке $x \in \mathbb{R}_{+}$существует конечный предел $\bar{j}_{\alpha}(f)(x)=\lim _{n \rightarrow+\infty}\left(f * W_{n}^{\alpha}\right)(x)$, то число $\bar{j}_{\alpha}(f)(x)$ назовем двоичным интегралом (ДИ) порядка $\alpha$ функции $f$ в точке $x$.

ТЕОрема 8. Ядро Уолша $\psi(\circ, y) \equiv \psi_{y}(\circ)($ см. (2)) имеет ДП и ДИ порядка $\alpha>0$ в любой точке $x \in \mathbb{R}_{+}$, причем $\bar{d}^{(\alpha)}\left(\psi_{0}\right)(x)=\bar{j}_{\alpha}\left(\psi_{0}\right)(x) \equiv 0$ на $\mathbb{R}_{+}$, а для $y>0, x \in \mathbb{R}_{+}$справедливы равенства $\bar{d}^{(\alpha)}\left(\psi_{y}\right)(x)=(h(y))^{-\alpha} \psi_{y}(x) u$ $\bar{j}_{\alpha}\left(\psi_{y}\right)(x)=(h(y))^{\alpha} \psi_{y}(x)$.

Для $\alpha=1$ первая часть этой теоремы, касающаяся двоичной производной, фактически доказана в [7], однако определение двоичной производной первого порядка в [7] несколько отличается от нашего, причем функции заданы на локально компактной двоичной группе $K_{2}$ (см. выше разд. 1 ).

ЗАмЕчАНИЕ 2 . Нетрудно доказать, что функция $\varphi=\mathrm{X}_{[0,1)}$ имеет ДП порядка $\alpha>0$ в каждой точке $x \in \mathbb{R}_{+}$, причем $\bar{d}^{(\alpha)}(\varphi)(x)=\left(2^{\alpha+1}-1\right)^{-1}$ для $x \in[0,1)$ и $\bar{d}^{(\alpha)}(\varphi)(x)=-\left(1-2^{-\alpha}\right) \sum_{k=1}^{+\infty} 2^{-k(\alpha+1)} \mathrm{X}_{\left[0,2^{k}\right)}(x)$ для $x \geqslant 1$. Эта же функция имеет ДИ порядка $\alpha \in(0,1)$ в каждой точке $x \in \mathbb{R}_{+}$и ни в одной точке $x \in \mathbb{R}_{+}$ не имеет ДИ порядка $\alpha \geqslant 1$.

Сравнивая эти результаты с формулами $\bar{d}^{(\alpha)}\left(\psi_{0}\right)(x) \equiv 0$ и $\bar{j}_{\alpha}\left(\psi_{0}\right)(x) \equiv 0$, где $\psi_{0}(x) \equiv 1$ на $\mathbb{R}_{+}$(см. теорему 8$)$, можно заключить, что наличие в данной точке двоичной производной или двоичного интеграла порядка $\alpha>0$ не является локальным свойством функции.

Как показывают следующие две теоремы, определения 2 и 3 согласованы друг с другом.

ТЕОрема 9. Если бункция $f \in L\left(\mathbb{R}_{+}\right)$при некотором $\alpha>0$ удовлетворяет условию $h^{\alpha} \tilde{f} \in L\left(\mathbb{R}_{+}\right)$(или условию $\left.h^{-\alpha} \tilde{f} \in L\left(\mathbb{R}_{+}\right)\right)$, то она имеет ДИ $\bar{j}_{\alpha}(f)(x)$ (соответственно ДП $\left.\bar{d}^{(\alpha)}(f)(x)\right)$ почти всюоу $\left(\right.$ п.в.) на $\mathbb{R}_{+}$, причем $\bar{j}_{\alpha}(f)(x)=j_{\alpha}(f)(x)$ n.в. (соответственно $\bar{d}^{(\alpha)}(f)(x)=d^{(\alpha)}(f)(x)$ n.в.). При этом, согласно теореме $5, j_{\alpha}(f)(x)$ и $d^{(\alpha)}(f)(x)$ существуют всюду на $\mathbb{R}_{+}$.

Теорема 10. Каждая из функиий $a_{m, n}(x)=\psi\left(x, m 2^{-n}\right) \mathrm{X}_{\left[0,2^{n}\right)}(x)(m \in \mathbb{N}$, $n \in \mathbb{Z})$ имеет ДИ и ДП, а такэе МДИ и МДП порядка $\alpha>0$ в любой точке $x \in \mathbb{R}_{+}$, причем $\bar{j}_{\alpha}\left(a_{m, n}\right)(x)=j_{\alpha}\left(a_{m, n}\right)(x)=2^{-r \alpha} a_{m, n}(x)$ и $\bar{d}^{(\alpha)}\left(a_{m, n}\right)(x)=$ $d^{(\alpha)}\left(a_{m, n}\right)(x)=2^{r \alpha} a_{m, n}(x)$, где челое число $r=r(m, n)$ однозначно определяется вложением $\left[m 2^{-n},(m+1) 2^{-n}\right) \subset\left[2^{r}, 2^{r+1}\right)$.

Следующая теорема является двоичным аналогом классических теорем о дифференцировании и интегрировании преобразования Фурье.

Tеорема 11. Пусть $\alpha>0 u f, h^{-\alpha} f \in L\left(\mathbb{R}_{+}\right)\left(f, h^{\alpha} f \in L\left(\mathbb{R}_{+}\right)\right)$. Тогда в каждой точке $x \in \mathbb{R}_{+}$преобразование Фуръе-Уолиа $\tilde{f}$ имеет двоичную производную (двоичный интеграл) порядка $\alpha$, причем $\bar{d}^{(\alpha)}(\tilde{f})(x)=\widehat{\left(\widehat{h^{-\alpha} f}\right)(x)}($ coответственно $\left.\bar{j}_{\alpha}(\tilde{f})(x)=\widehat{\left(h^{\alpha} f\right)}(x)\right)$. 
При условии, что $f(x), x f(x) \in L\left(\mathbb{R}_{+}\right)$, равенство $\left.(\tilde{f})^{[1]}(x)=\widetilde{(t f(t)}\right)(x)$ для всех $x \in \mathbb{R}_{+}$доказал Пал [8].

\section{ЛитературА}

1. Butzer P. L., Wagner H. J. Appl. Analysis, 3, No. 1, 29-46 (1973). 2. Butzer P. L., Wagner H. J. In: Proc. Symp. Naval Res. Laboratory, Washington, D.C., April 18-20, 1973, pp. 75-81. 3. Wagner J. H. In: Proc. Symp. Theory and applications of Walsh functions, Hatfield Polytechnic, UK, 1975, pp. 101-129. 4. Butzer P. L., Wagner H. J. Analysis Math., 1, 171-196 (1975). 5. Onneweer C. W. Analysis Math., 3, 119-130 (1977). 6. Pal J., Simon P. Acta Math. Acad. Sci. Hung., 29, 155-164 (1977). 7. Onneweer C. W. Appl. Analysis, 9, 267-278 (1979). 8. Pal J. Annales Univ. Sci. Budapest. Sect. Math., 18, 49-54 (1975). 9. Голубов Б. И. Матем. сб., 193, №4, 37-60 (2002). 10. Fine N. J. Trans. Amer. Math. Soc., 69, 66-77 (1950). 11. Schipp F., Wade W. R., Simon P. Walsh series. An introduction to dyadic harmonic analysis. Akademiai Kiado, Budapest, 1990. 12. Голубов Б. И., Ефимов А. В., Сквориов В. А. Ряды и преобразования Уолша. Теория и применения. Наука, М., 1987. 13. Onneweer $C$. W. Trans. Amer. Math. Soc., 258, 923-931 (1980). 14. Голубов Б. И. Изв. РАН, Сер. матем., 67, №1, 33-58 (2003). 15. Голубов Б. И. Изв. РАН, Сер. матем., 65, №3, 3-14 (2001). 16. Pal J., Schipp F. Annales Univ. Sci. Budapest. Sect. Computatorica, 8, 91-108 (1987). 17. Schipp F. Mathematica Balkanica, 4, 541-546 (1974). 18. Wagner J. H. Ein Differential- und Integralkalkül in der Walsh-Fourier Analysis mit Anwendungen. Westdeutscher Verlag, Köln-Opladen, 1974. 19. Pal J., Schipp F. In: Theory and applications of Gibbs derivatives. Proc. First Intern. Workshop on Gibbs Derivatives. Math. Inst., Beograd, 1989, pp. 103-113.

Московский инженерно-физический институт e-mail: golubov@mail.mipt.ru

Поступило в редакцию 28 августа 2003 г.

\section{УДК 517.5}

\section{Демпфированные осцилляторные интегралы и проблема ограниченности максимальных операторов}

(c) 2005. И. А. Икромов

1. История и мотивы проблемы. Одним из классических результатов действительного анализа является так называемая максимальная теорема И. М. Стейна о сферических средних в $\mathbb{R}^{n+1}(n \geqslant 2)$ [10]. Позднее аналог теоремы Стейна для плоского случая был доказан Дж. Бургеном в работе [4]. Эти результаты стали отправной точкой для изучения различных классов максимальных операторов, связанных с подмногообразиями евклидова пространства. В данной работе рассматриваются максимальные операторы $M$, определенные соотношением

$$
M g(x)=\sup _{t>0}\left|\int_{S} g(x-t y) \psi(y) d \sigma(y)\right|,
$$

где $S \subset \mathbb{R}^{n+1}$ - гладкая гиперповерхность, $\psi$ - фиксированная неотрицательная функция с компактным носителем, $d \sigma$ - индуцированная лебегова мера на $S$ и $g \in C_{0}^{\infty}\left(\mathbb{R}^{n+1}\right)$. 